Relations industrielles

Industrial Relations

\title{
Workers's Control, A Reader on Labor and Social Change, Edited by Gerry Hunnius, G. David Gardon and John Case, New York, Random House, 1973, 493 pp.
}

\section{Gilles Laflamme}

\section{Volume 29, numéro 1, 1974}

URI : https://id.erudit.org/iderudit/028491ar

DOI : https://doi.org/10.7202/028491ar

Aller au sommaire du numéro

Éditeur(s)

Département des relations industrielles de l'Université Laval

ISSN

0034-379X (imprimé)

1703-8138 (numérique)

Découvrir la revue

Citer ce compte rendu

Laflamme, G. (1974). Compte rendu de [Workers's Control, A Reader on Labor and Social Change, Edited by Gerry Hunnius, G. David Gardon and John Case, New York, Random House, 1973, 493 pp.] Relations industrielles / Industrial Relations, 29(1), 228-229. https://doi.org/10.7202/028491ar

Tous droits réservés @ Département des relations industrielles de l'Université Laval, 1974
Ce document est protégé par la loi sur le droit d'auteur. L'utilisation des services d’Érudit (y compris la reproduction) est assujettie à sa politique d'utilisation que vous pouvez consulter en ligne.

https://apropos.erudit.org/fr/usagers/politique-dutilisation/ 
par lecteur optique relié à un ordinaentreprises. Par contre, l'informatique teur, implique des dépenses que ne peuvent encourir les petites et les moyennes n'est pas le seul moyen pour établir un décompte des heures et la paie des employés. Il existe d'autres instruments de calcul qui rendent possible l'extension de cette pratique à la petite et moyenne entreprise.

L'implantation de l'horaire flexible à l'échelle de la société industrielle créerait une occasion de revalorisation de l'individu au travail par un accroissement de responsabilités et par un élargissement des choix personnels, et cela, à un moment où les exigences de la technologie continuent à influencer les comportements en milieu de travail. C'est une réflexion globale qui se dégage de l'ensemble de l'ouvrage et c'est ce qui, à mon sens, en constitue l'aspect le plus intéressant.

Laurent BELANGER

Université Laval

Workers' Control, A Reader on Labor and Social Change, Edited by Gerry Hunnius, G. David Gardon and John Case, New York, Random House, 1973, 493 pp.

Le sujet qu'abordent les auteurs, «Le contrôle ouvrier», est loin d'être un sujet nouveau bien qu'il n'ait jamais été tellement à la mode au Canada et aux Etats-Unis.

En effet, le problème de la démocratisation des rapports du travail au moyen d'une gestion de l'entreprise par les travailleurs a toujours été au centre des théories socialistes et c'est une demande qui s'inscrit dans la tradition du mouvement ouvrier international.

L'intérêt que nous portons à ce livre est d'autant plus grand que peu de travaux portant sur le contrôle ouvrier ont été publiés ou sont disponibles en Amérique du Nord. De plus, les auteurs ont su éviter le danger d'offrir une option qui pourrait nous apparaître de peu d'intérêt eu égard à la valeur de notre système de relations du travail.
C'est pourquoi, ce livre, très bien conçu, comprend d'abord une critique sévère de la négociation collective dont "le rôle aujourd'hui est de fournir un cadre institutionnel rigide à la lutte des classes ». On fait ressortir les limites de ce système institutionnel. On porte également un jugement sévère sur le syndicalisme américain «qui sert les intérêts des grandes firmes » et qui contribue à intégrer la force ouvrière au système. Nous retrouvons sur cette question d'excellents articles de Stanley Aronowitz et de Daniel Bell.

Après une critique du système de négociation collective nord-américain, les auteurs présentent certains modèles contemporains de participation et d'autogestion. Ainsi les idées de « contrôle ouvrier 》 perdent ce qu'elles pouvaient avoir d'obscures pour s'offrir à nous comme une réalité. La perspective critique est toujours présente aussi bien dans l'analyse du système yougoslave que suédois que dans celle du modèle de cogestion allemand, lequel est perçu par Helmut Schauer comme une « rationalisation des relations sociales existantes » et non comme une «suppression du pouvoir capitaliste $\gg$.

Et dans une dernière partie les auteurs présentent le «contrôle ouvrier» comme une des stratégies de changement. Le «contrôle ouvrier» ne saurait être perçu comme une fin en soi, ni comme une simple réforme industrielle. Il ne trouve sa vraie signification que s'il est placé dans «la perspective stratégique d'une révolution politique et sociale ». Les articles de André Gorz et de Ernest Mandel sont des plus intéressants sur cette question.

Il est nécessaire avant tout de retenir que le «contrôle ouvrier» se situe totalement en dehors des théories participationnistes actuelles et tel que présenté dans ce livre il est une revendication anticapitaliste. On voit rnal comment il pourrait être absorbé ou digéré par le système au même titre que des augmentations de salaires ou des avantages sociaux accrus. C'est tout le problème de la relation entre stratégie et fin qui est posé. 
Le mérite de ce livre pourrait être de faire renaître un débat autour de ce thème et de reposer le problème du contrôle syndical obtenu par la négociation collective, de la bureaucratisation du syndicalisme et de son intégration.

\section{Gilles LAFLAMME}

\section{Université Laval}

Manpower Programs in the Policy Mix, by Lloyd Ulman (ed.). Baltimore, The John Hopkins University Press, 1973, $166 \mathrm{pp}$.

Cet ouvrage présente cinq essais synthétiques sur les possibilités et le rốle de la politique de main-d'oeuvre au niveau macroéconomique. On y reprend le débat déjà amplement discuté sur le conflit d'arbitrage entre l'inflation et le chômage (courbe de Phillips). Les présentations de R. Gordon et de C. Holt reprennent en substance des points de vue qu'ils ont déjà soutenus dans des études récentes et qui portent sur l'analyse de facteurs structurels et frictionnels de l'emploi sur le marché du travail. La notion centrale de leurs thèses est celle de «segmentation» du marché du travail.

L'article de R. Meidner et R. Anderson à la fin du volume, sur la nature et l'impact des politiques sélectives de marché du travail pratiquées en Suède et qu'on retrouve dans le fameux modèle de Rehn, donne une illustration intéressante des actions sectorielles préconisées par R.A. Gordon, en tenant compte toutefois des différences contextuelles tant politiques et sociales qu'économiques. S. Levitan considère la portée des programmes actuels américains de main-d'oeuvre à déplacer la courbe de Phillips vers la gauche, tandis que L. Thurrow s'arrête sur les effets redistributifs de tels programmes.

Les discussions au sujet de la validité de la relation originale ainsi que des versions revues et corrigées de la Courbe de Phillips n'ont pas fini de soulever des divergences de point de vue. Bien qu'à prime abord il semble qu'on trouve des contrastes entre les divers auteurs, ceux-ci résultent davantage des conceptions sur les rapports entre le chômage et l'inflation et des problèmes envisagés.

Gordon et Holt fondent leurs observations sur une théorie «frictionnelle élargie du chômage.

Gordon offre une typologie du chômage basée sur les conditions de mobilité interne et externe propre à chaque segment, qui nie toute homogénéité du marché et en appelle à des mesures correctrices distinctes axées sur la réduction des taux de roulement et de la durée de la recherche d'emploi.

Ces considérations reposent toutefois sur un indice de dispersion du chômage qui n'a pour le moment qu'une valeur descriptive, tout comme le rapport vacances d'emplois/taux de chômage et salaire monétaire utilisé par Holt et ses collègues chez qui l'analyse porte sur le long terme et ne couvre partiellement qu'un aspect (cost-markup) de la théorie inflationniste des prix. Gordon constate un accroissement de la dispersion entre les taux de chômage par occupation ainsi que par sexe et âge, incidemment relié aux changements dans la composition de la main-d'oeuvre active. On ne peut toutefois $\mathrm{y}$ voir une relation entre l'inflation et le chômage. Holt introduit à cet effet un modèle empirique qui peut compléter l'indice de Gordon. Suivent certaines recommandations pertinentes pour l'avenir des politiques de maind'oeuvre.

Avec Thurrow, c'est par une critique acerbe des objectifs de croissance, de stabilisation et de plein emploi qu'est abordé le rôle de la politique de maind'oeuvre. Pour l'auteur de «Human Capital $\gg$, l'échec des politiques fiscales et monétaires relève principalement d'obstacles créés par l'émergence de pouvoirs institutionnels sur les marchés des produits comme des facteurs; les groupes défavorisés (pauvres, victimes de conflits sociaux) absorbent continuellement ces échecs. Aussi, la politique de main-d'oeuvre, si elle se veut un moyen d'atteindre l'équité sociale, ne doit surtout pas reprendre les mêmes chemins.

Certains resteront surpris de la façon un peu trop expéditive avec laquelle l'au- 